Copyright is owned by the Author of the thesis. Permission is given for a copy to be downloaded by an individual for the purpose of research and private study only. The thesis may not be reproduced elsewhere without the permission of the Author. 


\section{Biopolymer Networks: Image Analysis, Reconstruction and Modeling}

A thesis presented in partial fulfilment of the requirements

for the degree of

Doctor of Philosophy

in

Physics

at Massey University, Manawatū,

New Zealand

Pablo Hernandez-Cerdan

2018

MASSEY UNIVERSITY

TE KUNENGA KI PŪREHUROA

UNIVERSITY OF NEW ZEALAND 
It's not about the end, it's all about the path.

¡Buen camino!. 


\section{Abstract}

The aim of this work is to extract the architecture of biopolymer networks from 3D images. This is motivated to further understand non-affine regimes, found during network formation and in low-density biopolymer networks, where the geometry of a network has a fundamental role in defining its mechanical properties.

Firstly, developed image analysis tools were extended to 3D and contributed to high-performance open-source libraries for image analysis. These developments in isotropic wavelets will help in extracting realistic networks by removing spurious noise generated during image acquisition.

Secondly, images of biopolymer gels from transmission electron microscopy (TEM), were used to reliably extract the network architecture. The imaged material was also studied with small-angle x-ray scattering (SAXS), and the comparison showed a strong agreement for network-size features.

Thirdly, spatial graphs were extracted from the image. A one-to-one map is provided between image and graph, keeping all the geometric information from the image. This then opened the door to using analytical tools from the complex networks field to characterize images.

Finally, statistical distributions extracted from three graph properties were used to reconstruct a completely in-silico network using a simulated annealing technique to generate new networks. This can be used as a computational exploration tool of how network behavior depends on network architecture. 


\section{Acknowledgements}

A special thank to my supervisor Bill Williams whose support, openness, and vision of science is encouraging. As Brad would say, I will be forever indebted to your supervision and I add: and from all the stuff that I have learned from you beyond academia, specially your humane values and the love for dumplings. Thanks to Brad Mansel, Leif Lundin and Andrew Leis for the collaboration comparing scattering and imaging techniques, Allan Raudsepp for providing the confocal microscopy images for actin, and to all my group mates from the Biophysics and Soft Matter group, for all the shared moments, and how easy they made my adaptation to a new country. Finally, I am grateful to the MacDiarmid Institute for constantly providing the enrichment opportunity to meet other peer students and researchers, and to the staff of the Institute of Fundamental Sciences for its kindness dealing with all sort of issues.

In the personal side, I would like to thank my family, from who I have inherited, directly and indirectly, all the principles I stand for and made me realize how privileged I am to have them. And Martica! Without you here in New Zealand this quest would have been impossible, or faster, who knows, but way less enjoyable for sure. It has been a pleasure habernos re-encontrado por kiwi-land. And even we got married, in English!!, crazy, but made me feel extremely fortunate. Thanks really for all your support.

This research was supported by the MacDiarmid Institute, the Riddet Institute, and Massey University, New Zealand. 


\section{Contents}

$\begin{array}{ll}\text { Abstract } & \text { ii }\end{array}$

Acknowledgements $\quad$ iii

Contents iv

List of Figures vii

List of Publications $\quad$ x

Abbreviations $\quad$ xi

1 Introduction $\quad 1$

1.1 Soft matter and biopolymers . . . . . . . . . . . . . . . . . . . . . . . . . . . 1

1.2 Semiflexible single chains: WLC model . . . . . . . . . . . . . . . . . . . . . . . 2

1.3 Networks of semiflexible chains: . . . . . . . . . . . . . . . . . 5

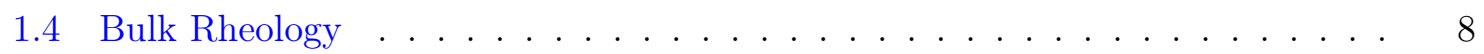

1.4 Linear viscoelasticity . . . . . . . . . . . . . . . . . . . . . 8

1.4.2 Nonlinear behaviour: strain stiffening . . . . . . . . . . . . . . . . . . 9

1.5 Network Structure . . . . . . . . . . . . . . . . . . . . . . . . . . . . . . . . . 11

1.5.1 Mikado Networks . . . . . . . . . . . . . . . . . . . . . . . . . . . . . . . . 11

1.5 .2 Lattice Models . . . . . . . . . . . . . . . . . . . . . . . 12

1.5 .3 Eight-chain Models . . . . . . . . . . . . . . . . . . . . . . . . . . . . . . . 13

1.6 Motivation . . . . . . . . . . . . . . . . . . . . . . . . . . . . . . 14

2 Isotropic and Steerable Wavelets in N Dimensions. A multiresolution $\begin{array}{ll}\text { analysis framework } & 16\end{array}$

2.1 Motivation . . . . . . . . . . . . . . . . . . . . . 16

2.2 Abstract . . . . . . . . . . . . . . . . . . . . 16

2.3 Wavelet Multiresolution Analysis . . . . . . . . . . . . . . . . . 17

2.3 .1 Introduction . . . . . . . . . . . . . . . . . 17

2.3.2 Motivation: spatial and frequency resolution . . . . . . . . . . . . 18

2.3.3 Wavelet transformation . . . . . . . . . . . . . . . . . . . . 19

2.3.4 Wavelet Pyramid . . . . . . . . . . . . . . . . . . . . . . . . . 21

2.3 .5 Isotropic wavelets . . . . . . . . . . . . . . . . . . . . . . . . . . . . . . 22

2.4 Riesz Transform . . . . . . . . . . . . . . . . . . . . . . . . . . . . . . . . . . . . . . . 25

2.4.1 Monogenic Signal . . . . . . . . . . . . . . . . . 26 
2.4.2 Generalized Riesz Transform _. . . . . . . . . . . . . . . . . 27

2.4.3 Generalized Steerable Framework . . . . . . . . . . . . . . . . 27

2.5 Implementation Details . . . . . . . . . . . . . . . . . . . . . 28

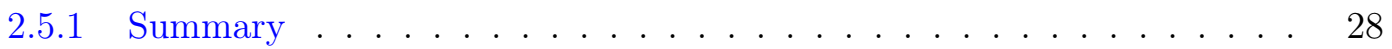

2.5 .2 Frequency Iterators . . . . . . . . . . . . . . . . . . . . . 29

2.5.3 Wavelet Transform . . . . . . . . . . . . . . . . . . . 30

2.5.4 Riesz Transform . . . . . . . . . . . . . . . . . . . . . . . . . . . . . . . . 31

2.5.5 Structure Tensor . . . . . . . . . . . . . . . . . . . 31

2.5 .6 Phase Analysis . . . . . . . . . . . . . . . . . . . . . . 32

2.6 A guided example: . . . . . . . . . . . . . . . . . . . . . . . . . . . 32

2.6.1 Input in the frequency domain. . . . . . . . . . . . . . . . . . . . . . . . . . . . 33

2.6.2 Choosing an isotropic wavelet . . . . . . . . . . . . . 33

2.6.3 Forward / Analysis . . . . . . . . . . . . . . . . . . . 33

2.6.4 Inverse / Reconstruction . . . . . . . . . . . . . . . . . . . . . 34

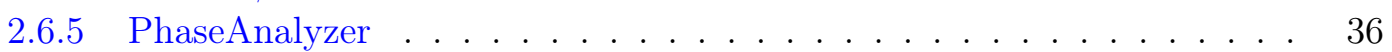

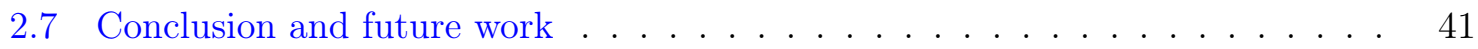

2.8 Acknowledgements . . . . . . . . . . . . . . . . . . . . . 42

3 Validation of Transmission Electron-Microscopy Imaging: Comparison with SAXS

3.1 Motivation . . . . . . . . . . . . . . . . . . . 43

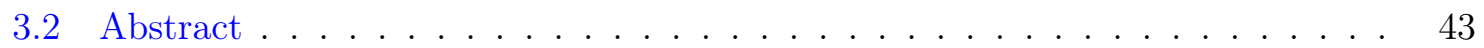

3.3 Introduction . . . . . . . . . . . . . . . . . . . . . . . 44

3.4 Materials and methods . . . . . . . . . . . . . . . . . . . . . . . . . . . 45

3.5 Results and Discussion . . . . . . . . . . . . . . . . . . . . . . . . . . . . . . . . . . . . . .

3.5.1 Qualitative Structural Features . . . . . . . . . . . . . . . . 49

3.5.2 Consistency Between Techniques at Different Lengthscales . . . . . . . 50

3.5.3 Image Processing . . . . . . . . . . . . . . . . . . . . . . 52

3.5.4 Extracting Persistence Lengths . . . . . . . . . . . . . . . . . 54

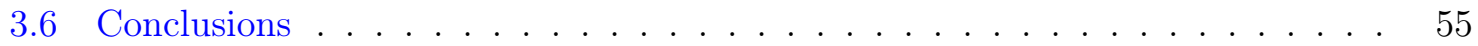

4 Spatial Graph Extraction: Analysis of microscopy images to obtain network $\begin{array}{ll}\text { architecture } & 58\end{array}$

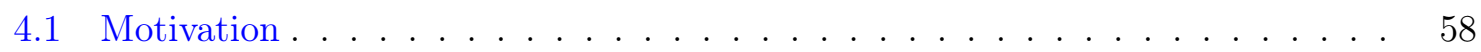

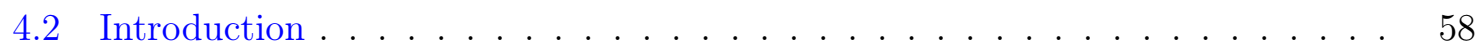

4.2 .1 Notation . . . . . . . . . . . . . . . . . . . . . . . 60

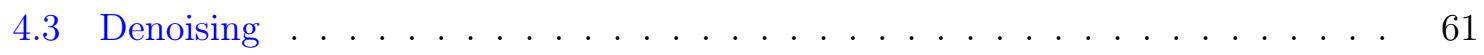

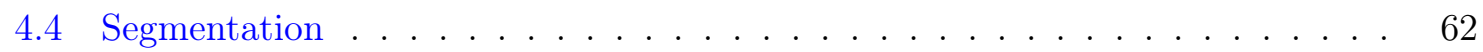

4.4 Binarization . . . . . . . . . . . . . . . . . 63

4.4.1.1 Region Growing Segmentation . . . . . . . . . . . . . 64

4.4 Hole Filling . . . . . . . . . . . . . . . . . . . . . . 65

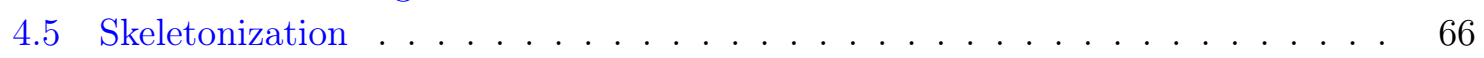

4.5 .1 Distance maps . . . . . . . . . . . . . . . . . . . 68

4.5.2 Critical Kernels Framework . . . . . . . . . . . . . . . . . 68

4.6 Spatial Graph Extraction . . . . . . . . . . . . . . . . . . . . 72

4.7 Statistical distributions of graph properties . . . . . . . . . . . . . . . . . 74

4.8 Testing existing thinning algorithms . . . . . . . . . . . . . 75 
4.8.1 FIbeR Extraction -FIRE- . . . . . . . . . . . . . . . . . 75

4.8.1.1 Results in FIRE . . . . . . . . . . . . . . 77

4.8.2 Avizo: XSkeleton . . . . . . . . . . . . . . . . . . . . . . . 79

4.8.2.1 Avizo results: . . . . . . . . . . . . . . . . . 81

4.9 Application of the Network Analysis Pipeline to Different Biopolymer Networks. 84

4.9.1 Confocal Light Microscopy: Actin . . . . . . . . . . . . . . . . . . . . 84

4.9 .2 TEM preparation . . . . . . . . . . . . . . . . . . . . . 90

4.9.3 Transmission Electron Microscopy: Carrageenan _. . . . . . . . . . . 91

4.9.4 Transmission Electron Microscopy: Pectin . . . . . . . . . . . . . . . . 94

4.10 Conclusions . . . . . . . . . . . . . . . . . . . . . . . 97

5 Reconstructing networks from statistical distributions $\quad 99$

5.1 Motivation . . . . . . . . . . . . . . . . . . . . . . . . 99

5.2 Methods . . . . . . . . . . . . . . . . . . . . . . . . 99

5.3 Euclidean Graph Generation Algorithm . . . . . . . . . . . . . . . . 102

5.4 Results . . . . . . . . . . . . . . . . . . . . . . . . . 103

6 Conclusions and Future Work 106

6.1 Scope of Thesis . . . . . . . . . . . . . . . . . . . 106

6.2 Summary . . . . . . . . . . . . . . . . . . . . 106

6.3 Conclusions . . . . . . . . . . . . . . . . . . . . . . . . 108

6.4 Future work . . . . . . . . . . . . . . . . . . . . . . 109

6.4 .1 Network Formation . . . . . . . . . . . . . . . . . . . . . . . . 109

6.4.2 Long time behaviour: network quakes and aging . . . . . . . . . . . . 109

6.4 .3 ITKBoostGraph . . . . . . . . . . . . . . . . . . . . . . 110

6.4 .4 Complex Networks Tools . . . . . . . . . . . . . . . . . . . . . . . 110

6.4.5 Public Database . . . . . . . . . . . . . . . . . . . . . . 110

A Publications and Open Source Contributions 111

A.1 Open Source Contributions . . . . . . . . . . . . . . . . . . . . . . . . 111

A.1.1 ITKIsotropicWavelets . . . . . . . . . . . . . . . . . . . . 111

A.1.2 Radial Intensity FFT . . . . . . . . . . . . . . . . . . . . . . 111

A.1.3 FFT Radial Intensity . . . . . . . . . . . . . . . . . . . . . . . . . . . . . . . . . . . . . . .

A.1.4 DGtal: Critical Kernels Framework . . . . . . . . . . . . . . . . . 112

A.1.5 Spatial Graph Extraction . . . . . . . . . . . . . . . . . . 113

A.1.6 Open Science . . . . . . . . . . . . . . . . . . . . . . . 113

$\begin{array}{ll}\text { Glossary } & 114\end{array}$

$\begin{array}{ll}\text { References } & 116\end{array}$ 


\section{List of Figures}

1.1 Optical Tweezers . . . . . . . . . . . . . . . . . . 5

1.2 Force extension curve: CEWLC . . . . . . . . . . . . . . . 6

1.3 Strain-stiffening in semiflexible polymers . . . . . . . . . . . . . . . . 9

1.4 Affine and non-affine deformations . . . . . . . . . . . . . . . 10

1.5 Affine and non-affine phases . . . . . . . . . . . . . . . 11

1.6 Mikado network used as a starting scaffold for dynamics simulations, from Ref. [31]. . . . . . . . . . . . . . . . . . . . . . . . 12

1.7 Unit cell of a face-centered-cubic lattice, from Ref. [40] . . . . . . . . . . . . 13

1.8 Eight-chain model under shear, from left to right: initial state, a rotation, and a simple shear, from Ref. Palmer and Boyce [30] . . . . . . . . . . . . . . . 14

2.1 The changes over time/space of a signal are not captured by the FFT. . . . . 18

2.2 Simultaneous spatial and frequency resolution of different transformations. $\Delta t, \Delta f \quad 19$

2.3 Forward 2.3a and Inverse wavelet 2.3b two-level pyramid with two high pass sub-bands. . . . . . . . . . . . . . . . . . . . 21

2.4 Forward wavelet pyramid with a classic two level filter bank and only one sub-band, HP is the high pass filter, and LP low pass . . . . . . . . .

2.5 Tiling of the frequency domain by isotropic wavelets when the dilation factor is 2. All the wavelets fulfill the conditions from Proposition 2.3. The mother wavelets are represented at $i=0 . \ldots \ldots \ldots \ldots$

2.6 Shape of SubBands when HighPassSubBands $=5$, sub-bands increase the frequency resolution, but can generate extra artifacts in the spatial domain due to the sharp frequency cut-offs. . . . . . . . . . . . . . . . .

2.7 The original image looks like a regular check-board, but it isn't. Pixels in the regions A and B have the same intensity value (129), however our vision system performs local phase analysis that allows us to treat A,B regions as different, keeping a global checker-board structure. 2.7b uses a non-linear map of intensity-color to enhance the irregular checker board structure. . . . . . . .

2.8 (Results of the phase analysis (with soft threshold) for different number of scales in the wavelet pyramid. The input image is a checker-board of size $512 x 512$ ).

2.9 Using six scales (Level: 6), results for different number of high frequency sub-bands. . . . . . . . . . . . . . . . . . . . . .

2.10 The optical illusion generated by 2.10a the Hermann grid is generated by the local phase analysis of our vision. $2.10 \mathrm{~b}$ is the result of a phase analysis with the Monogenic signal for a wavelet pyramid of 8 levels and only one high pass sub band. 2.10c is the same pyramid but with 10 high pass bands to increase the frequency resolution. Both results use Simoncelli wavelet, but there is not much difference using Held, or Vow mother wavelets. . . . . . . . . . . . . . 
2.11 Biopolymer network (actin) 3D image enhanced and denoised using phase analysis with the Monogenic signal, with a wavelet pyramid of 4 levels and 4 high pass sub-bands using Simoncelli isotropic wavelet.

3.1 The Graphical User Interface (GUI) of the image analysis software developed herein. A non-interactive interface is also provided for batch processing, along with python scripts for plotting and manipulation of the generated data. . . .

3.2 Montages of TEM images obtained from different polysaccharides, as described in the text.

3.3 SAXS results for the three samples shown in the TEM micrographs of figure 3.2, in the absence of TEM sample preparation. Black lines signify power law fitting, as is described in section 3.1 of the text. . . . . . . . . . . . .

3.4 Comparison of data obtained from TEM best practice and SAXS. Good agreement can be seen in the large network scale (at low $q \mathrm{~s}$ ). The shaded area shows the upper $q$ range (smaller than $20 \mathrm{~nm}$ or equivalently 25 pixels). The top $\mathrm{x}$-axis shows the corresponding real-space length scale, where $d=2 \pi / q$. .

3.5 Results obtained when applying two widely-used de-noising techniques: total variation regularization (TV) and multi-scale wavelet denoising using a BLSGSM algorithm. . . . . . . . . . . . . . . . .

3.6 Visual comparison of the de-noised pectin TEM images (Fig 3.2a). (a) shows a region from the unmodified original image, (b): de-noised image using a total variation method [78] with $\lambda=0.15$, (c) shows the same region after applying a multi-scale wavelet approach using the BLS-GSM algorithm [58] with $\sigma=28.4$. Only high-frequency regions are affected by de-noising algorithms, as reflected in the generated I-q plots shown in Fig 3.5a. . . . . . . . . . . . . .

3.7 A TEM micrograph with FibreApp tracking output shown in blue. The insert shows the internal contour length vs mean-square end-to-end distance calculated from the corresponding tracking parameters. . . . . . . . . . . . . .

4.1 Visualization of a Spatial Graph (4.1a) and the corresponding adjacency list (4.1b). The graph is represented in blue, formed by nodes (voxels with id) and edges connecting them. The voxel positions (in brackets in 4.1a) of nodes and edge points are stored as labels in the adjacency list. . . . . . . . . . .

4.2 Pre-processing the image (actin): denoising with wavelets and total variation methods. . . . . . . . . . . . . . . . . . 62

4.3 Different threshold values on networks . . . . . . . . . . . .

4.4 Thin image of a pectin network after skeletonization performed with the critical kernels framework (see subsection 4.9.4) and visualized with a 3D Viewer. The skeleton keeps the topology of the original object, is thin, centered, and robust against rough and noisy boundaries. . . . . . . . . . . .

4.5 Distance map - Actin . . . . . . . . . . . . . . . . . . . 68

4.6 Asymmetric thinning algorithm with persistence, from Ref. [91] . . . . . . .

4.7 Asymmetric thinning of a volume with different parameters. (a): Ultimate skeleton, only voxels that conserve topology are kept. (b): keeps 1-isthmus as part of the skeleton. (c): 1 -isthmus with persistence parameter $p=10 \ldots$.

4.8 Skeletonization . . . . . . . . . . . . . . . . .

4.9 Extracting the raw graph from the thin image and post processed to remove the largest edges between tri-connected nodes with degree 3. Numbers show the number of edges (degree) of each node. 
4.10 Spatial Graph where vertices with degree 2 are deleted, and its position are stored as edge points in the new edges. Numbers in circles represent degree of each node. . . . . . . . . . . . . . . . . . . . . . . . . . . . . . . . . . . . 74

4.11 FIRE algorithm - Find LMP . . . . . . . . . . . . . . . . . . . . . . 77

4.12 Fire: Step by step for $T_{P}=0.12 \ldots \ldots \ldots \ldots \ldots \ldots$

4.13 Distributions of length and degree in FIRE . . . . . . . . . . . . . . 79

4.14 Avizo image: Actin network visualization and workspace . . . . . . . . . . . 80

4.15 Distributions of length, degree, and cosines with Avizo for actin with $\mathrm{T}=30, \mathrm{P}=582$

4.16 Distributions of length, degree, and cosines with Avizo for actin with $\mathrm{T}=21, \mathrm{P}=583$

4.17 Actin spatial graph extraction steps. . . . . . . . . . . . . . . 88

4.18 Statistical distributions (PDF) of actin network. Computed properties of the graph are represented in histograms with bins and normalized by area to obtain the PDF. Orange line shows the function with parameters obtained from a non-linear least squares fit to the data. Green lines show the same function with fixed parameters: $4.18 \mathrm{a}$ : mean degree $(Z)$ of the network, $4.18 \mathrm{c}$ : mean $\left(\mu_{l}\right)$ and standard deviation $\left(s_{l}\right)$ of logarithmic end-to-end distances, $4.18 \mathrm{~d}$ mean $\left(\mu_{l}\right)$ and standard deviation $\left(s_{l}\right)$ of logarithmic contour lengths. Histogram bins: end-to-end distances (30), direction cosines (21) contour lengths (30) . . . . 90

4.19 Potassium carrageenan spatial graph extraction steps. . . . . . . . . . . . . 93

4.20 Statistical distributions (PDF) of Potassium Carrageenan network. Computed properties of the graph are represented in histograms with bins and normalized by area to obtain the PDF. Orange line shows the function with parameters obtained from a non-linear least squares fit to the data. Green lines show the same function with fixed parameters: 4.20a: mean degree $(Z)$ of the network, $4.20 \mathrm{c}$ : mean $\left(\mu_{l}\right)$ and standard deviation $\left(s_{l}\right)$ of logarithmic end-to-end distances, 4.20d mean $\left(\mu_{l}\right)$ and standard deviation $\left(s_{l}\right)$ of logarithmic contour lengths. Histogram bins: end-to-end distances (50), direction cosines (21) contour lengths $(50) \ldots \ldots \ldots \ldots \ldots \ldots \ldots \ldots \ldots 4 \ldots \ldots \ldots \ldots$

4.21 Pectin spatial graph extraction steps. . . . . . . . . . . . . . 96

4.22 Statistical distributions (PDF) of Pectin network. Computed properties of the graph are represented in histograms with bins and normalized by area to obtain the PDF. Orange line shows the function with parameters obtained from a non-linear least squares fit to the data. Green lines show the same function with fixed parameters: $4.22 \mathrm{a}$ : mean degree $(Z)$ of the network, 4.22c: mean $\left(\mu_{l}\right)$ and standard deviation $\left(s_{l}\right)$ of logarithmic end-to-end distances, $4.22 \mathrm{~d}$ mean $\left(\mu_{l}\right)$ and standard deviation $\left(s_{l}\right)$ of logarithmic contour lengths. Histogram bins: end-to-end distances (50), direction cosines (21) contour lengths (50) . . . . .

5.1 Collagen distributions . . . . . . . . . . . . . . . . . . . 100

5.2 Testing with theoretical collagen data . . . . . . . . . . . . . . 104

5.3 Collagen: comparing target and simulated distributions . . . . . . . . . 105

6.1 Scheme of the thesis . . . . . . . . . . . . . . . . . 108 


\section{List of Publications}

[1] Pablo Hernandez-Cerdan. "Isotropic and Steerable Wavelets in N Dimensions. A Multiresolution Analysis Framework for ITK." In: arXiv:1710.01103 [cs] (Oct. 2017). arXiv: 1710.01103 [cs].

[2] Pablo Hernandez-Cerdan, Bradley W. Mansel, Andrew Leis, Leif Lundin, and Martin A.K. Williams. "Structural Analysis of Polysaccharide Networks by Transmission Electron Microscopy: Comparison with Small-Angle X-Ray Scattering." In: Biomacromolecules (Jan. 2018). ISSN: 1525-7797. DOI: 10.1021/acs. biomac.7b01773.

[3] Hina Shah, Pablo Hernandez, Francois Budin, Deepak Chittajallu, Jean-Baptiste Vimort, Rick Walter, André Mol, Asma Khan, and Beatriz Paniagua. "Automatic Quantification Framework to Detect Cracks in Teeth." In: Medical Imaging 2018: Biomedical Applications in Molecular, Structural, and Functional Imaging. Vol. 10578. International Society for Optics and Photonics, Mar. 2018, 105781K. DOI: 10.1117/ 12. 2293603. 


\section{Abbreviations}

AFM Atomic force microscopy.

BGL Boost Graph Library. c ++ library.

CEWLC Clickable extensible wormlike chain.

DFT Discrete Fourier Transform, representation of data in frequency domain.

DGtal Digital Geometry Tools and Algorithms. c ++ library.

EtE End to end.

EWLC Extensible wormlike chain.

FE Force extension (curve).

FFT Fast Fourier Transform, specific algorithm of DFT.

ITK Insight ToolKit. Image Analysis c ++ library specialized to work with ND images.

LAOS Large amplitude oscillatory shear (rheology).

LMP Local maximum point.

MRA Multiresolution framework.

MSD Mean square displacement.

NP Nucleation point.

PME Pectin-methylesterase.

SAXS Small angle X-ray scattering .

SEM Scanning electron microscopy.

TEM Transmission electron microscopy.

TV Total Variation (regularization, denoising method).

WLC Wormlike chain. 